\title{
Precision medicine program for epithelial ovarian carcinoma (EOC): First year experience at Clínica Universidad de Navarra (CUN)
}

Luisa Sánchez-Lorenzo1,a, Iñigo Espinosa²,a, Enrique Chacón,b, Ana Patiño-García4,b, Jaime Espinós ${ }^{1, a}$, José Manuel Aramendía1,b, Teresa Castellanos3,a, Daniel Vázquez,a, José A. Mínguez,b, Gorka AlkortaAramburu4,b, Miguel A. Idoate2,b, Juan Luis Alcázar3,b, Luis Chiva ${ }^{3, a}$ and Antonio González-Martín',a

1.Medical Oncology Department, 2. Pathology Department, 3. Gynecology Department. 4. CIMA-LAB Diagnostics

a. Clínica Universidad de Navarra, Madrid, Spain b. Clínica Universidad de Navarra, Pamplona, Spain

\section{Background}

Next Generation Sequencing (NGS) technology enables for the simultaneous study of multiple gene alterations in the patient's tumor with potential implications for genetic risk assessment, prognosis, and therapy.

\section{Methods}

- CUN opened a new hospital in Madrid in January 2018. During the first year, new patients (pts) with EOC at both CUN hospitals (Madrid and Pamplona) were offered to perform a NGS-based tumor testing.

- Local test consisted in Oncomine Comprehensive Assay (161 gene panel, ThermoFisher Scientific) and external test in Foundation Medicine (Foundation Medicine, Inc., Cambridge, MA) (FM).

- This is a descriptive analysis of the rate of implementation, the molecular alterations found, and the therapeutic implications derived.

\section{Results}

- 104 new pts were evaluated from January $1^{\text {st }}$ until December 31st.

- 5 (4.80\%) LOGSOC, 81 (77.89\%) HGSOC, 11 (10.58\%) CCC, 6 (5.77\%) endometrioid, 1 $(0.96 \%)$ Mucinous. 44 pts with primary disease and 60 pts with recurrent disease.

- All but two patients were tested with Oncomine with a median turn-around time of 14 days. Table shows the rate of patients tested according to histology and context of disease.

- sBRCA mutation was detected by Oncomine in 6/23 primary diagnosis (26.1\%) and 2 pts received olaparib maintenance after SOLO-1 publication (both gBRCAwt).
- In the recurrent setting, 5/16 pts (31.25\%) had at least one actionable mutation with available matched therapy:

- Pathogenic mutations: MSH2, MLH6

- Pathogenic mutation: 2 patients with ATM

- PI3KCA mutation

- ERBB2 amplification

- and 3 pts received matched therapy (18.76\%)

- MSH2: Pembrolizumab

- MLH6: Pembrolizumab

- ATM: 1 included in a clinical trial with Avelumab + Talazoparib

- In addition, non-BRCA genes potentially associated to hereditary EOC were detected in 4 pts (1 with RAD50, 1 RAD51, and 2 ATM).

\section{Conclusions}

- Integration of a precision medicine program with NGS tumor testing in EOC was feasible in routine practice in a private university hospital, with an optimal turn-around time.

- This is an ongoing program and the rate of tumor testing should improve in subsequent years as far as all the physicians become more familiar with the precision medicine program.

\begin{tabular}{l|c|c|c|}
\multicolumn{1}{c}{ Subtype } & $\mathbf{N}$ & Tested in FL & $\begin{array}{c}\text { Tested in } \\
\text { recurrence }\end{array}$ \\
\hline LGSOC & 5 & $2 / 2(100 \%)$ & $1 / 3(33.33 \%)$ \\
\hline HGSOC & 81 & $17 / 34(50 \%)$ & $14 / 47(29,78 \%)$ \\
\hline Endometrioid & 6 & $1 / 2(50 \%)$ & $0 / 4(0 \%)$ \\
\hline CCC & 11 & $3 / 6(50 \%)$ & $1 / 5(20 \%)$ \\
\hline Mucinous & 1 & $0 / 0$ & $0 / 1(0 \%)$ \\
\hline TOTAL & 104 & $23 / 44(52.57 \%)$ & $16 / 60(26.67 \%)$ \\
\hline
\end{tabular}

Table 1. Results 